\title{
BOUNDARY BEHAVIOR OF HARMONIC FORMS ON A RANK ONE SYMMETRIC SPACE
}

\author{
BY
}

\author{
AROLDO KAPLAN(1) AND ROBERT PUTZ(2)
}

\begin{abstract}
We study the boundary behavior of 1-forms on a rank-one symmetric space $M$ satisfying the equations $d \omega=0=\delta \omega$; the role of boundary is played by a nilpotent (Iwasawa) group $\bar{N}$ of isometries of $M$. For forms satisfying certain $H^{p}$ integrability conditions, we obtain the existence of boundary values in an appropriate sense, characterize these boundary values by means of fractional and singular integral operators on the group $\bar{N}$, and exhibit explicit isomorphisms between $H^{P}$ spaces of forms on $M$ and the ordinary $L^{P}$ spaces of functions on the group $\bar{N}$.
\end{abstract}

Let $M$ be a Riemannian manifold and let $\delta$ be the adjoint of the exterior differential $d$ on $M$. The equation $d \omega=0=\delta \omega$ can be considered as a generalization of the classical Cauchy-Riemann equations. Their solutions were studied by Stein and Weiss in the case $M=\mathbf{R}^{n} \times \mathbf{R}^{+}$with the euclidean metric (conjugate systems of harmonic functions) [9], by Korányi and Vági in the case when $M$ is a euclidean ball in $\mathbf{R}^{n}$ [7], and by Coifman and Weiss in the case $M=G \times \mathbf{R}^{+}, G$ being a compact Lie group with the bi-invariant metric [1].

In this paper we consider the case when $M$ is a noncompact symmetric space of rank one, define $\mathbf{H}^{p}$ spaces of 1 -forms satisfying the above equations and study their boundary behavior. The role of boundary is played by a nilpotent group $\bar{N}$ of isometries of $M$, which is the Cartan-conjugate of $N$ in a fixed Iwasawa decomposition $G=K A N$ of the connected group of isometries of $M$ [5].

A right action of the solvable group $\bar{S}=A \bar{N}$ induces a decomposition of the tangent bundle of $M$ along the $A$ and $\bar{N}$-directions ("vertical" and "horizontal" directions, by analogy with the upper half plane). It is shown that if a form $\omega$ is in certain $\mathbf{H}^{p}$ classes, its components along these directions have boundary values and that, moreover, the boundary values of the horizontal components can be obtained from the boundary values of the vertical

Received by the editors February 2, 1976.

AMS (MOS) subject classifications (1970). Primary 43A85, 53C35; Secondary 35H05.

(') Partially supported by NSF grant GP29703A3.

(2) Partially supported by NSF grant GP28448. 
component by means of fractional and singular integral operators on the group $\bar{N}$ (the Riesz transforms). The kernels of these operators, introduced in $\$ 2$, arise as left-invariant derivatives of a fundamental solution of a secondorder hypoelliptic operator on the group $\bar{N}$. We are then able to recover a form $\omega$ in $\mathbf{H}^{p}$ from the boundary value of its vertical component, and to establish in this manner canonical isomorphisms between the $\mathbf{H}^{p}$ spaces of forms and the ordinary $L^{p}$ spaces on the group $\bar{N}$.

1. A class of vector fields on $M$. The symmetric space $M$ can be expressed as a homogeneous space $M=G / K$ where $G$ is a semisimple group of isometries of $M$ and $K$ is a maximal compact subgroup of $G$. Let $g$, denote the Lie algebras of $G$ and $K, B$ the Killing form of $\mathfrak{g}$, and $\mathfrak{p}$ the orthogonal complement of $f$ in $g$ relative to $B$. If $\pi: G \rightarrow G / K$ denotes the canonical projection, its differential at the identity, $\pi_{*}$, identifies the subspace $\mathfrak{p}$ of $g$ with $T_{0}(M)$, the tangent space of $M$ at the origin $o=\pi(e)$, and the invariant metric $g$ on $M$ can be chosen so that $g_{0}$ corresponds to the restriction of $B$ to $\mathfrak{p} \times \mathfrak{p}$ under the above identification.

Let $a$ be a maximal abelian subspace of $p, \Delta \subseteq a^{*}$ the corresponding system of (restricted) roots, and for each $\alpha \in \Delta$ let $g_{\alpha}$ denote the corresponding root space. Let $\Delta_{+}$denote the system of positive roots relative to the choice of a fixed lexicographic ordering in $a^{*}$; if $\alpha \in \Delta_{+}$, we write $\bar{g}_{\alpha}=g_{-\alpha}$. Then $\mathfrak{n}=\sum_{\alpha \in \Delta_{+}} \mathrm{g}_{\alpha}, \overline{\mathfrak{n}}=\sum_{\alpha \in \Delta_{+}} \overline{\mathrm{g}}_{\alpha}$ are nilpotent subalgebras of $\mathrm{g}$, and if $A, N, \bar{N}$, are the connected subgroups of $G$ with Lie algebras $\mathfrak{a}, \mathfrak{n}, \overline{\mathfrak{n}}$, one has the Iwasawa decompositions $G=K A N$ and $G=\bar{N} A K$.

Now, $\bar{S}=\bar{N} A$ is a solvable subgroup of $G$ and the above decomposition shows that every $p \in M$ can be uniquely written as $p=s \cdot o(s \in \bar{S})$. We can therefore define a right action $\tau$ of $\bar{S}$ on $M$ by letting

$$
\tau(s)\left(s^{\prime} \cdot o\right)=s^{\prime} s \cdot o \quad\left(s, s^{\prime} \in \bar{S}\right) .
$$

For each $X \in \bar{\Xi}=\overline{\mathfrak{n}}+$ a define a vector field $\tilde{X}$ on $M$ by letting

$$
\tilde{X}_{n \cdot \cdot o}=\tau_{*}\left(\operatorname{Ad}\left(a^{-1}\right) X\right)_{n a \cdot o} \quad(n \in \bar{N}, a \in A) .
$$

Now let $X$ be any nonzero element of $\bar{s}$; for each fixed $a \in A$, the vector field $\tau_{*}\left(\operatorname{Ad}\left(a^{-1}\right) X\right)$ never vanishes on $M$, because $\operatorname{Ad}\left(a^{-1}\right)$ is an automorphism of $\bar{\Xi}$ and $\tau$ is a free action; therefore, the same is true of the vector field $\tilde{X}$. It then follows that $X \rightarrow \tilde{X}$ maps a basis of $\overline{\bar{s}}$ into a global frame of vector fields on $M$.

We shall now compute the Lie brackets and the inner products between vector fields induced in the above manner. Fix $a \in A$; the mapping $\Phi_{a}: n$ $\rightarrow n a \cdot o$ is a diffeomorphism from $\bar{N}$ onto the submanifold $\bar{N} a \cdot o \subseteq M$, and if $X \in \overline{\mathfrak{n}}$, then $\left.\tilde{X}_{n a \cdot o}=\left(\Phi_{a_{*}} X\right)\right)_{n a \cdot . \circ}$. Therefore the vector fields $\tilde{X},(X \in \overline{\mathfrak{n}})$ are tangent to the submanifolds $\overline{N a} \cdot o$, and the map $X \rightarrow \tilde{X}$ is a Lie algebra 
homomorphism from $\bar{n}$ into the Lie algebra of all smooth vector fields on $M$. On the other hand, if $n_{0} \in \bar{N}, a_{0} \in A$, the diffeomorphisms of $\bar{S}$ given by $n a \rightarrow n n_{0} a$ and $n a \rightarrow n a_{0} a$ commute with each other; it follows that if $H \in \mathfrak{a}$, then $[\tilde{H}, \tilde{X}]=0$ for every $X \in \bar{\xi}$.

Now, let $X \in \bar{s}$; the induced vector field $\tilde{X}$ can be expressed as $\tilde{X}_{\text {na.o }}$ $=(n a)_{*}\left(\pi_{* e}\left(\operatorname{Ad}\left(a^{-1}\right) X\right)\right)$, and from the invariance of the Riemannian metric $g$, it follows that

$$
g_{n a \cdot o}(\tilde{X}, \tilde{Y})=g_{o}\left(\pi_{* e}\left(\operatorname{Ad}\left(a^{-1}\right) X\right), \pi_{* e}\left(\operatorname{Ad}\left(a^{-1}\right) Y\right)\right) \quad(X, Y \in \bar{s})
$$

Let $\theta$ denote the Cartan involution associated to the decomposition $\mathfrak{g}=\mathfrak{t}+\mathfrak{p}$ and let $(\cdot, \cdot)$ denote the inner product $-B(\cdot, \theta \cdot)$ on $g$. Then

$$
\begin{aligned}
g_{0}\left(\pi_{* e}\left(Z_{1}\right), \pi_{* e}\left(Z_{2}\right)\right) & =B\left(\frac{1}{2}\left(Z_{1}-\theta Z_{1}\right), \frac{1}{2}\left(Z_{2}-\theta Z_{2}\right)\right) \\
& =\frac{1}{2}\left(Z_{1}, Z_{2}\right)-\frac{1}{2}\left(Z_{1}, \theta Z_{2}\right) .
\end{aligned}
$$

Now using (2) and (3), together with the fact that the subalgebras $\mathfrak{a}, \mathfrak{n}$, and $\overline{\mathfrak{n}}=\theta \mathfrak{n}$ are mutually orthogonal relative to the inner product $(\cdot, \cdot)$, one gets the following expression for the inner product of two arbitrary vector fields induced from $\overline{\mathrm{s}}$ :

$$
g_{n a \cdot o}\left(\left(H_{1}+X_{1}\right)^{\tilde{2}},\left(H_{2}+X_{2}\right)^{\tilde{}}\right)=\left(H_{1}, H_{2}\right)+\frac{1}{2}\left(\operatorname{Ad}\left(a^{-1}\right) X_{1}, \operatorname{Ad}\left(a^{-1}\right) X_{2}\right),
$$

where $H_{1}, H_{2} \in \mathfrak{a}, X_{1}, X_{2} \in \overline{\mathrm{n}}$.

In particular, if $X$ is a root vector corresponding to the root $-\alpha\left(\alpha \in \Delta_{+}\right)$ and $H$ is in a, then the induced vector fields $\tilde{H}$ and $\tilde{X}$ are orthogonal, $\tilde{H}$ has constant length equal to $(H, H)^{1 / 2}$, and the length of $\tilde{X}$ is given by

$$
g_{n a \cdot o}(\tilde{X}, \tilde{X})=\frac{1}{2} e^{2 \alpha(\log a)}(X, X) .
$$

In $\$ 3$ it will be useful to have at our disposal a formula for the codifferential (or "divergence") $\delta \omega$ of a 1 -form $\omega$ in terms of the vector fields introduced above. First of all, if $Y_{1}, \ldots, Y_{n}$ is an orthonormal frame defined on an open subset of a Riemannian manifold $(V, g)$, and $\omega$ is a 1 -form on $V$, then $\delta \omega=\sum_{i}\left(Y_{i} \omega\left(Y_{i}\right)-t\left(Y_{i}\right) \omega\left(Y_{i}\right)\right)$, where $t\left(Y_{i}\right)=\sum_{j} g\left(\left[Y_{i}, Y_{j}\right], Y_{j}\right)$; this expression is easily derived from any of the standard definitions of the operator $\delta$.

Now choose a basis $\left\{H_{i}, X_{\alpha, j}\right\}$ of the Lie algebra $\overline{\bar{s}}$, such that $H_{i} \in \mathfrak{a}, X_{\alpha, j}$ $\in \overline{\mathfrak{g}}_{\alpha}$, and orthonormal with respect to the inner product $(\cdot, \cdot)=-B(\cdot, \theta \cdot)$. Letting $e^{\alpha}$ be the function on $M$ whose value at the point $n a \cdot o(n \in \bar{N}, a$ $\in A)$ is $e^{\alpha(\log a)}$, from definition (1) it follows that $\tilde{H}_{i}=\tau_{*}\left(H_{i}\right)$ and $\tilde{X}_{\alpha, j}$ $=e^{\alpha} \tau_{*}\left(X_{\alpha, j}\right)$. Therefore (5) implies that $\left\{\tau_{*}\left(H_{i}\right), \sqrt{2} \tau_{*}\left(X_{\alpha, j}\right)\right\}$ is an orthonormal frame on $M$. Since $\tau_{*}$ is a Lie algebra homomorphism, the relations $\left[H_{i}, X_{\alpha, j}\right]=-\alpha\left(H_{i}\right) X_{\alpha, j},\left[X_{\alpha, j}, X_{\beta, k}\right] \in \overline{\mathrm{g}}_{\alpha+\beta}$ imply that, relative to this ortho- 
normal frame, $t\left(\tau_{*}\left(X_{\alpha, j}\right)\right)=0$ for all $\alpha, j$, and $t\left(\tau_{*}\left(H_{i}\right)\right)=-\Sigma_{\alpha \in \Delta_{+}} m_{\alpha} \alpha\left(H_{i}\right)=$ $-2 \rho\left(H_{i}\right)$ (where as usual we put $m_{\alpha}=\operatorname{dim} g_{\alpha}, \rho=\frac{1}{2} \sum_{\alpha \in \Delta_{+}} m_{\alpha} \alpha$ ). Hence

$$
\delta \omega=\sum_{i} \tau_{*}\left(H_{i}\right) \omega\left(\tau_{*}\left(H_{i}\right)\right)+2 \sum_{\alpha} \sum_{j} \tau_{*}\left(X_{\alpha, j}\right) \omega\left(\tau_{*}\left(X_{\alpha, j}\right)\right)+2 \sum_{i} \rho\left(H_{i}\right) \omega\left(\tilde{H}_{i}\right) .
$$

Now $\sum_{i} \rho\left(H_{i}\right) H_{i}=H_{\rho}$, the vector dual to $\rho$; since $\tau_{*}\left(X_{\alpha_{j}}\right)=e^{-\alpha} \tilde{X}_{\alpha, j}$ and the vector field $\tilde{X}_{\alpha, j}$ annihilates the function $e^{-\alpha}$, one obtains

$$
\delta \omega=\sum_{i} \tilde{H}_{i} \omega\left(\tilde{H}_{i}\right)+2 \omega\left(\tilde{H}_{\rho}\right)+2 \sum_{\alpha \in \Delta_{+}} \sum_{j} e^{-2 \alpha} \tilde{X}_{\alpha, j} \omega\left(\tilde{X}_{\alpha, j}\right) .
$$

Now assume that the rank of $M$ is one. One can then choose a positive root $\alpha$ such that either $\Delta_{+}=\{\alpha\}$ or $\Delta_{+}=\{\alpha, 2 \alpha\}$. Let $H_{0} \in \mathfrak{a}$ be the vector such that $\alpha\left(H_{0}\right)=1$; then

$$
\left(H_{0}, H_{0}\right)=B\left(H_{0}, H_{0}\right)=\operatorname{trace}\left(\operatorname{ad} H_{0}\right)^{2}=2\left(m_{\alpha}+4 m_{2 \alpha}\right) .
$$

Now set $\eta=\left(16\left(m_{\alpha}+4 m_{2 \alpha}\right)\right)^{-1}$ and $m=m_{\alpha}+2 m_{2 \alpha}$; then the vector $(8 \eta)^{1 / 2} H_{0}$ has unit length, and since $\left(H_{\rho}, H_{0}\right)=\rho\left(H_{0}\right)=\frac{1}{2}\left(m_{\alpha}+2 m_{2 \alpha}\right)=$ $m / 2$, one obtains $H_{\rho}=4 \eta m H_{0}$. Let $\left\{X_{i}\right\}_{i=1}^{m_{m}}$ and $\left\{Y_{j}\right\}_{j=1}^{m_{2 a}}$ be orthonormal bases of $\overline{\mathrm{g}}_{\alpha}$ and $\overline{\mathrm{g}}_{2 \alpha}$, respectively; then (6) now implies

$$
\begin{aligned}
\delta \omega= & 8 \eta \tilde{H}_{0} \omega\left(\tilde{H}_{0}\right)+8 \eta m \omega\left(\tilde{H}_{0}\right)+2 e^{-2 \alpha} \sum_{i} \tilde{X}_{i} \omega\left(\tilde{X}_{i}\right) \\
& +2 e^{-4 \alpha} \sum_{j} \tilde{Y}_{j} \omega\left(\tilde{Y}_{j}\right) .
\end{aligned}
$$

For notational convenience we replace the vector field $\tilde{H}_{0}$ (which has constant length) by the vector field $\tilde{W}=e^{2 \alpha} \tilde{H}_{0}$, which grows on the order of the vector fields induced by the elements of $\bar{g}_{2 \alpha}$. Since $\tilde{H}_{0}\left(e^{-2 \alpha}\right)=-2 e^{-2 \alpha}$ and $\tilde{H}_{0} \omega\left(\tilde{H}_{0}\right)=e^{-4 \alpha} \tilde{W} \omega(\tilde{W})-2 e^{-2 \alpha} \omega(\tilde{W})$, we obtain from (7):

LEMMA 1 . In the notation above,

$$
\begin{aligned}
\frac{1}{8 \eta} e^{2 \alpha} \delta \omega= & e^{-2 \alpha} \tilde{W} \omega(\tilde{W})+(m-2) \omega(\tilde{W})+\frac{1}{4 \eta} \sum_{i} \tilde{X}_{i} \omega\left(\tilde{X}_{i}\right) \\
& +\frac{1}{4 \eta} e^{-2 \alpha} \sum_{j} \tilde{Y}_{j} \omega\left(\tilde{Y_{j}}\right) .
\end{aligned}
$$

2. Riesz transforms on $\bar{N}$. This section is concerned with some analysis on the nilpotent group $\bar{N}$. In particular, we will construct some integral operators on this group which will play a central role in the characterization of the boundary values of harmonic forms on the space $M$.

From now on we assume that the rank of $M$ is one; thus, $\overline{\mathfrak{n}}=\overline{\mathrm{g}}_{\alpha} \oplus \overline{\mathrm{g}}_{2 \alpha}$. Let $\left\{X_{i}\right\}, 1 \leqslant i \leqslant m_{\alpha}$, be an orthonormal basis of $\bar{g}_{\alpha}$, and define 


$$
L=\sum_{i} X_{i}^{2}
$$

then $L$ is a differential operator on $\bar{N}$ which is independent of the choice of orthonormal basis of $g_{\alpha}$. Note that $L$ is not elliptic, unless $\bar{g}_{2 \alpha}=(0)$ (in this case, which occurs when $M$ is a real hyperbolic space, $\bar{N}$ is isomorphic to $\mathbf{R}^{n}$ and $L$ becomes the standard Laplacian); however, since any basis of $\bar{g}_{\alpha}$ constitutes a system of generators for the Lie algebra $\overline{\mathfrak{n}}$, a result of Hörmander [4] implies that $L$ is always hypoelliptic. Our first objective will be to obtain an explicit fundamental solution for this operator.

For $X \in \mathfrak{g}$, set $|X|=(X, X)^{1 / 2}$. The following lemma holds without restriction on the rank of $M$.

Lemma 2. Let $\alpha$ be a restricted root, $X \in \overline{\mathrm{g}}_{\alpha}$, and $Y \in \overline{\mathrm{g}}_{2 \alpha}$. Then $\left.\| Y, \theta X\right]$ $=\sqrt{2}|\alpha||X||Y|$. If $\left\{X_{i}\right\}, 1 \leqslant i \leqslant m_{\alpha}$, is an orthonormal basis of $\bar{g}_{\alpha}$, then

$$
\sum_{i=1}^{m_{\alpha}}\left|\left[X, X_{i}\right]\right|^{2}=2 m_{2 \alpha}|\alpha|^{2}|X|^{2} .
$$

Proof. We have

$$
\|\left.[Y, \theta X]\right|^{2}=-B([Y, \theta X],[\theta Y, X])=B([[Y, \theta X], X], \theta Y) .
$$

Since $\left[\overline{\mathrm{g}}_{\alpha}, \overline{\mathrm{g}}_{2 \alpha}\right]=(0),[[Y, \theta X], X]=-[Y,[X, \theta X]]$ by Jacobi's identity. Now $[X, \theta X]=-B(X, \theta X) H_{\alpha}=|X|^{2} H_{\alpha}$ and $\left[Y, H_{\alpha}\right]=2 \alpha\left(H_{\alpha}\right) Y=2|\alpha|^{2} Y$. Therefore

$$
|[Y, \theta X]|^{2}=-2|\alpha|^{2}|X|^{2} B(Y, \theta Y)=2|\alpha|^{2}|X|^{2}|Y|^{2},
$$

proving the first identity.

Now let $\left\{X_{i}\right\}, 1 \leqslant i \leqslant m_{\alpha}$, be as above, and choose an orthonormal basis $\left\{Y_{j}\right\}, 1 \leqslant j \leqslant m_{2 \alpha}$, of $\bar{g}_{2 \alpha}$. Then

$$
\left|\left[X, X_{i}\right]\right|^{2}=\sum_{j=1}^{m_{2 \alpha}}\left(Y_{j},\left[X, X_{i}\right]\right)^{2}=\sum_{j=1}^{m_{2 \alpha}}\left(\left[Y_{j}, \theta X\right], X_{i}\right)^{2} ;
$$

adding over $i=1, \ldots, m_{\alpha}$, one gets

$$
\sum_{i=1}^{m_{\alpha}}\left|\left[X, X_{i}\right]\right|^{2}=\sum_{j=1}^{m_{2 \alpha}}\left|\left[Y_{j}, \theta X\right]\right|^{2} .
$$

But the first part of the lemma shows that every term in the last sum is equal to $2|\alpha|^{2}|X|^{2}$, finishing the proof.

Any element $n \in \bar{N}$ can be written uniquely as $n=\exp (X+Y)$, with $X \in \overline{\mathfrak{g}}_{\alpha}, Y \in \overline{\mathfrak{g}}_{2 \alpha}$. A function $F$ on $\bar{N}$ will be called biradial if there is a function $f(u, v)$ of two real variables such that 


$$
F(\exp (X+Y))=f\left(|X|^{2},|Y|^{2}\right) .
$$

Lemma 3. Let $F$ be a smooth biradial function on $\bar{N}, F(\exp (X+Y))$ $=f\left(|X|^{2},|Y|^{2}\right)=f(u, v)$; let $X^{\prime} \in \overline{\mathrm{g}}_{\alpha}, Y^{\prime} \in \overline{\mathrm{g}}_{2 \alpha}$ and let $L$ be the operator defined by (8). Then, for $n=\exp (X+Y)$,

$$
\begin{aligned}
\left(X^{\prime} F\right)(n)= & 2\left(X^{\prime}, X\right) \partial f / \partial u+\left(\left[X, X^{\prime}\right], Y\right) \partial f / \partial v, \\
\left(Y^{\prime} F\right)(n)= & 2\left(Y^{\prime}, Y\right) \partial f / \partial v \\
(L F)(n)= & 4|X|^{2} \partial^{2} f / \partial u^{2}+2|\alpha|^{2}|X|^{2}|Y|^{2} \partial^{2} f / \partial v^{2} \\
& +2 m_{\alpha} \partial f / \partial u+m_{2 \alpha}|\alpha|^{2}|X|^{2} \partial f / \partial v .
\end{aligned}
$$

Proof. For $t \in \mathbf{R}$, one has

$$
\exp (X+Y) \exp t X^{\prime}=\exp \left(X+t X^{\prime}+Y+\frac{1}{2} t\left[X, X^{\prime}\right]\right) .
$$

Therefore

$$
F\left(n \exp t X^{\prime}\right)=f\left(\left|X+t X^{\prime}\right|^{2},\left|Y+\frac{1}{2} t\left[X, X^{\prime}\right]\right|^{2}\right)
$$

and

$$
\frac{d}{d t} F\left(n \exp t X^{\prime}\right)=2\left(\left(X, X^{\prime}\right)+t\left|X^{\prime}\right|^{2}\right) \frac{\partial f}{\partial u}+\left(\left(Y,\left[X, X^{\prime}\right]\right)+\frac{1}{2} t\left|\left[X, X^{\prime}\right]\right|^{2}\right) \frac{\partial f}{\partial v} .
$$

Therefore

$$
\left(X^{\prime} F\right)(n)=\left.\frac{d}{d t} F\left(n \exp t X^{\prime}\right)\right|_{t=0}=2\left(X^{\prime}, X\right) \frac{\partial f}{\partial u}+\left(\left[X, X^{\prime}\right], Y\right) \frac{\partial f}{\partial v},
$$

showing (i); also

$$
\begin{aligned}
\left(X^{\prime 2} F\right)(n) & =\left.\frac{d^{2}}{d t^{2}} F\left(n \exp t X^{\prime}\right)\right|_{t=0} \\
& =4\left(X, X^{\prime}\right)^{2} \frac{\partial^{2} f}{\partial u^{2}}+\left(Y,\left[X, X^{\prime}\right]\right)^{2} \frac{\partial^{2} f}{\partial v^{2}}+4\left(X, X^{\prime}\right)\left(Y,\left[X, X^{\prime}\right]\right) \frac{\partial^{2} f}{\partial u \partial v} \\
& +2\left|X^{\prime}\right|^{2}(\partial f / \partial u)+\frac{1}{2}\left|\left[X, X^{\prime}\right]\right|^{2}(\partial f / \partial v) .
\end{aligned}
$$

Now, if $\left\{X_{i}\right\}, 1 \leqslant i \leqslant m_{\alpha}$, is an orthonormal basis of $\overline{\mathrm{g}}_{\alpha}$, one has $\Sigma_{i}\left(X, X_{i}\right)^{2}$ $=|X|^{2}, \Sigma_{i}\left|X_{i}\right|^{2}=m_{\alpha}, \Sigma_{i}\left(X, X_{i}\right)\left(Y,\left[X, X_{i}\right]\right)=(Y,[X, X])=0$; also, Lemma 2 implies 


$$
\begin{aligned}
\sum_{i}\left(Y,\left[X, X_{i}\right]\right)^{2} & =\sum_{i}\left([Y, \theta X], X_{i}\right)^{2}=|[Y, \theta X]|^{2} \\
& =2|\alpha|^{2}|X|^{2}|Y|^{2}
\end{aligned}
$$

and

$$
\sum_{i}\left|\left[X, X_{i}\right]\right|^{2}=2 m_{2 \alpha}|\alpha|^{2}|X|^{2}
$$

Letting $X^{\prime}=X_{i}$ in (9), it now follows that $(L F)(n)=\Sigma_{i}\left(X_{i}^{2} F\right)(n)$ is given by (iii). Since (ii) is clear, this proves the lemma.

For each $\varepsilon \geqslant 0$, define a function $\|n\|_{\varepsilon}$ on $\bar{N}$ by $\|n\|_{\varepsilon}=\eta\left(|X|^{2}+\varepsilon^{2}\right)^{2}$ $+|Y|^{2}$, where $n=\exp (X+Y), X \in \overline{\mathrm{g}}_{\alpha}, Y \in \overline{\mathrm{g}}_{2 \alpha}$, and $\eta$ denotes the constant $|\alpha|^{2} / 8=\left(16\left(m_{\alpha}+4 m_{2 \alpha}\right)\right)^{-1}$. One verifies that under the "dilations" of $\bar{N}$ induced by the group $A$,

$$
\left\|a n a^{-1}\right\|_{\varepsilon}=e^{-4 \alpha(\log a)}\|n\|_{\varepsilon^{\prime}}, \quad \varepsilon^{\prime}=e^{\alpha(\log a)} \varepsilon, \quad a \in A .
$$

In particular, $\|n\|_{0}=\eta|X|^{4}+|Y|^{2}$ is a "gauge" on $\bar{N}$, in the sense of Korányi and Vági [6], which satisfies the homogeneity condition

$$
\left\|a n a^{-1}\right\|_{0}=e^{-4 a(\log a)}\|n\|_{0}, \quad a \in A .
$$

From now on we shall assume that $m=m_{\alpha}+2 m_{2 \alpha}>2$; this excludes the cases when $M$ is the real hyperbolic space of dimension 2 or 3 . Now, set $k=\frac{1}{4}\left(m_{\alpha}+2 m_{2 \alpha}-2\right)=\frac{1}{4}(m-2)$; since $\operatorname{dim} \bar{N}=m_{\alpha}+m_{2 \alpha}$, the function $\|n\|_{\varepsilon}^{-k-1}$ is integrable on $\bar{N}$ for every $\varepsilon>0$; one can then introduce a constant $\beta$ by $\beta^{-1}=-4 k \eta m_{\alpha} \int_{N}\|n\|_{1}^{-k-1} d n$.

THEOREM 1. The function $G(n)=\beta\|n\|_{0}^{-k}$ is a fundamental solution for the operator $L$.

Proof. A straightforward application of Lemma 3 to the biradial function $\|n\|_{\varepsilon}^{-k}$ shows that

$$
L\left(\|n\|_{\varepsilon}^{-k}\right)=-4 k \eta m_{\alpha} \varepsilon^{2}\|n\|_{\varepsilon}^{-k-1} .
$$

In particular, $L G(n)=0$ for all $n \neq e$.

We claim now that $L\left(\beta\|n\|_{\varepsilon}^{-k}\right)$ is an approximate identity as $\varepsilon \rightarrow 0$. In fact, by (12) one has

$$
\int_{\bar{N}} L\left(\beta\|n\|_{\varepsilon}^{k}\right) d n=\left[\int_{\bar{N}}\|n\|_{1}^{-k-1} d n\right]^{-1} \int_{\bar{N}} \varepsilon^{2}\|n\|_{\varepsilon}^{-k-1} d n .
$$

But, via the change of variables $n \rightarrow a^{-1} n a$, with $a=\exp \varepsilon H_{0}, \alpha\left(H_{0}\right)=1$, formula (10) shows that 


$$
\int_{\Gamma} \varepsilon^{2}\|n\|_{\varepsilon}^{-k-1} d n=\int_{\Gamma}\|n\|_{1}^{-k-1} d n \quad(\varepsilon>0) .
$$

Therefore $L\left(\beta\|n\|_{\varepsilon}^{-k}\right)$ is a positive $L^{1}$-function on $\bar{N}$ with $L^{1}$-norm one. Moreover, the same change of variables shows that for any $\delta>0$,

$$
\begin{aligned}
\int_{\|n\|>\delta} L\left(\|n\|_{\varepsilon}^{-k}\right) d n & =-4 k m_{\alpha} \eta \int_{\|n\|>\delta} \varepsilon^{2}\|n\|_{\varepsilon}^{-k-1} d n \\
& =-4 k m_{\alpha} \eta \int_{\|n\|>\delta / \varepsilon^{4}}\|n\|_{1}^{-k-1} d n,
\end{aligned}
$$

so that $\lim _{\varepsilon \rightarrow 0} \int_{\|n\|>\delta} L\left(\|n\|_{\varepsilon}^{-k}\right) d n=0$.

Now, if $f$ is a continuous function on $\bar{N}$ with compact support, and $g$ is smooth, then $D(f * g)=f * D g$ for any left-invariant differential operator $D$ on $\bar{N}$, where the convolution is given by $f * g(n)=\int_{N} f\left(n_{1}\right) g\left(n^{-1}, n\right) d n_{1}$. Hence

$$
L(f * G)=\lim _{\varepsilon \rightarrow 0} L\left(f * \beta\|n\|_{\varepsilon}^{-k}\right)=\lim _{\varepsilon \rightarrow 0} f * L\left(\beta\|n\|_{\varepsilon}^{-k}\right)=f .
$$

This finishes the proof of the theorem.

REMARK. In the case when $\bar{N}$ is the Heisenberg group $(M=$ complex hyperbolic space) this fundamental solution was obtained by Folland [2].

Definition. For $Z \in \bar{n}$, the $Z$-Riesz kernel is the function $r_{Z}(n)=Z G(n)$, $n \neq e$.

A straightforward application of Lemma 3 gives the following expressions of the above kernels:

$$
\begin{aligned}
& r_{Z}(n)=-k \beta\|n\|_{0}^{-k-1}\left[4 \eta|X|^{2}(Z, X)+([X, Z], Y)\right] \text { for } Z \in \overline{\mathrm{g}}_{\alpha}, \text { and } \\
& r_{Z}(n)=-2 k \beta\|n\|_{0}^{-k-1}(Y, Z) \text { for } Z \in \overline{\mathrm{g}}_{2 \alpha} .
\end{aligned}
$$

Under the adjoint action of $A$ on $\bar{N}$, these kernels satisfy the homogeneity properties

$$
\begin{aligned}
& r_{Z}\left(a^{-1} n a\right)=e^{-(m-1) \alpha(\log a)} r_{Z}(n) \quad \text { for } Z \in \bar{g}_{\alpha}, \text { and } \\
& r_{Z}\left(a^{-1} n a\right)=e^{-m \alpha(\log a)} r_{Z}(n) \quad \text { for } \quad Z \in \overline{\mathrm{g}}_{2 \alpha} ;
\end{aligned}
$$

in the latter case, one also has $r_{Z}\left(n^{-1}\right)=-r_{Z}(n)$. Thus, the functions $r_{Z}$ can be considered as fractional integral kernels (for $Z \in \overline{\mathrm{g}}_{\alpha}$ ) and singular integral kernels (for $Z \in \bar{g}_{2 \alpha}$ ), and a standard argument gives

THEOREM 2. The convolution operators defined by $R_{Z} f=f * r_{Z}$ are:

(a) bounded operators from $L^{p}(\bar{N})$ into $L^{p}(\bar{N})$, for $1<p<\infty$ and $Z \in \overline{\mathrm{g}}_{2 \alpha}$,

(b) bounded operators from $L^{p}(\bar{N})$ into $L^{q}(\bar{N})$, for $1<p<m, 1 / q=1 / p$ $-1 / m$, and $Z \in \overline{\mathrm{g}}_{\alpha}$. 
For more general results on fractional and singular integral operators on nilpotent groups see Stein [8] and Korányi and Vági [6].

We also note that these Riesz transforms satisfy the formal properties

$$
\begin{array}{ll}
X \circ R_{Y}-Y \circ R_{X}=R_{[X, Y]} & (X, Y \in \overline{\mathfrak{n}}), \\
\sum_{i} X_{i} \circ R_{X_{i}}=\text { identity } & \left(\left\{X_{i}\right\} \text { orthonormal basis of } \overline{\mathrm{g}}_{\alpha}\right) .
\end{array}
$$

We finish this section with a technical result that will be needed later.

Lemma 4. If $F_{1}, F_{2}$ are biradial functions on $\bar{N}$, then $F_{1} * F_{2}=F_{2} * F_{1}$.

Proof. With $n=\exp (X+Y), n_{1}=\exp \left(X_{1}+Y_{1}\right)$ and $F_{i}(\exp (X+Y))$ $=f_{i}\left(|X|^{2},|Y|^{2}\right)$, one has

$$
\begin{aligned}
F_{1} * F_{2}\left(n_{1}\right) & =\int_{N} F_{1}(n) F_{2}\left(n^{-1} n_{1}\right) d n \\
& =\int f_{1}\left(|X|^{2},|Y|^{2}\right) f_{2}\left(\left|X_{1}-X\right|^{2},\left|Y_{1}-Y-\frac{1}{2}\left[X, X_{1}\right]\right|^{2}\right) d X d Y .
\end{aligned}
$$

Under the orthogonal change of variables $X \rightarrow X^{\prime}=2\left(\left(X, X_{1}\right) /\left|X_{1}\right|^{2}\right) X_{1}-X$, one has, $\left|X_{1}-X^{\prime}\right|=\left|X_{1}-X\right|$ and $\left[X, X_{1}\right]=-\left[X^{\prime}, X_{1}\right]$. Thus,

$$
\begin{aligned}
F_{1} * F_{2}\left(n_{1}\right) & =\int f_{1}\left(|X|^{2},|Y|^{2}\right) f_{2}\left(\left|X_{1}-X\right|^{2},\left|Y_{1}-Y+\frac{1}{2}\left[X, X_{1}\right]\right|^{2}\right) d X d Y \\
& =\int_{N} F_{1}(n) F_{2}\left(n n^{-1}\right) d n=F_{2} * F_{1}\left(n_{1}\right),
\end{aligned}
$$

proving the lemma.

3. Boundary values of harmonic forms. We regard the nilpotent group $\bar{N}$ as a boundary for the symmetric space $M$, as, for example, in [5]. A function $\Phi$ on $M$ is said to be uniformly in $L^{p}$ if there exists a constant $K$ such that $\int_{\bar{N}}|\Phi(n a \cdot o)|^{p} d n<K$ for all $a \in A$. If the family of functions $\left\{\Phi_{a}, a \in A\right\}$ on $\bar{N}$ given by $\Phi_{a}(n)=\Phi(n a \cdot o)$ converges to a function $\varphi$ on $\bar{N}$ as $a \rightarrow \infty$ (in $L^{p}$, uniformly, etc.), one simply says that $\Phi$ converges to $\varphi$ or that $\varphi$ is the boundary value of $\Phi$. The following facts are well known: Let $1<p<\infty$ and let $\Phi$ be a harmonic function on $M$ which is uniformly in $L^{p}$. Then $\Phi$ has a boundary value $\varphi \in L^{p}(\bar{N})$ to which it converges in $L^{p}$ and almost everywhere. The Poisson kernel is the function $P$ on $\bar{N} \times A$ defined by

$$
P(n, a)=P_{a}(n)=\exp \left\{-2 \rho\left(H\left(a^{-1} n a\right)-\log a\right)\right\}
$$

where for each $g \in G, H(g)$ is the unique element in a such that $g$ $=k \exp H(g) n(k \in K, n \in N)$. If $\varphi \in L^{p}(\bar{N})$ one defines the Poisson integral of $\varphi$ as the function $\Phi$ on $M$ given by 


$$
\Phi(n a \cdot o)=\varphi * P_{a}(n)=\int_{N} \varphi\left(n_{1}\right) P_{a}\left(n^{-1} n\right) d n_{1}
$$

then $\Phi$ is harmonic on $M$, it is uniformly in $L^{p}$, and its boundary value is the original function $\varphi$.

We shall now define $H^{p}$ spaces of differential 1 -forms. The need for different integrability conditions for different components will be apparent in Theorems 3 and 4.

Definition. For $1<p<m\left(m=m_{\alpha}+2 m_{2 \alpha}\right)$, let $q$ be given by $1 / q$ $=1 / p-1 / m$, and let $\mathbf{H}^{p}$ be the space of all 1 -forms on $M$ such that

(i) $d \omega=0=\delta \omega$.

(ii) The functions $\omega(\tilde{X})\left(X \in \overline{\mathrm{g}}_{\alpha}\right)$, are uniformly in $L^{q}$.

(iii) The functions $\omega(\tilde{W}), \omega(\tilde{Y})\left(Y \in \bar{g}_{2 \alpha}\right)$ and $\sum_{i} \tilde{X}_{i} \omega\left(\tilde{X}_{i}\right)\left(\left\{X_{i}\right\}\right.$ orthonormal basis of $\bar{g}_{\alpha}$ ) are uniformly in $L^{p}$.

REMARK. It should be pointed out that the components $\omega(\tilde{W})$ and $\omega(\tilde{X})$ are not harmonic (except when $X$ belongs to the center of $\bar{n}$, a fact that is used below). Although replacing these vector fields by the infinitesimal isometries induced by $\overline{\mathfrak{n}}$ would give harmonic components, such vector fields are inappropriate in the present context.

Our next objective is to associate a form $\omega_{f} \in \mathbf{H}^{p}$ to each function $f \in L^{p}(\bar{N})$. For this we will need a kernel $Q$ on $M$ defined by

$$
Q(n a \cdot o)=Q_{a}(n)=c \tilde{W}\left(P_{a} * G(n)\right)=c \tilde{W} \int_{\bar{N}} P_{a}(g) G\left(g^{-1} n\right) d g
$$

where $G$ is the fundamental solution for the operator $L$ introduced in $\$ 2$, and $c$ is a constant to be specified later.

Under conjugation by $A$, the kernel $Q$ satisfies the homogeneity property

$$
Q_{a^{\prime}}\left(a^{-1} n a\right)=e^{-m \alpha(\log a)} Q_{a^{\prime} a}(n) .
$$

In fact, for the Poisson kernel one has $P_{a^{\prime}}\left(a^{-1} n a\right)=e^{-m \alpha(\log a)} P_{a^{\prime} a}(n)$ and therefore, $\tilde{W} P_{a^{\prime}}\left(a^{-1} n a\right)=e^{-(m+2)} \tilde{W} P_{a^{\prime} a}(n)$; on the other hand, $G\left(a^{-1} n a\right)$ $=e^{-(m-2) \alpha(\log a)} G(n)$ so that (14) follows by the definition of $Q$ as the convolution of $\tilde{W} P_{a}$ with $G$.

LEMMA 5. For an appropriate choice of the constant c, the kernel $Q_{a}$ is an approximate identity on $\bar{N}$, that is:

(i) $Q_{a}(n)>0$ and $Q_{a} \in L^{1}(\bar{N})$,

(ii) $\int_{N} Q_{a}(n) d n=1$ for all $a \in A$,

(iii) $\lim _{t \rightarrow \infty} \int_{\bar{N}(\varepsilon)} Q_{a_{t}}(n) d n=1$ for all $\varepsilon>0$, where $a_{t}=\exp t H_{0}$ and $\bar{N}(\varepsilon)$ $=\{n \in \bar{N}:\|n\| \leqslant \varepsilon\}$.

Proof. The proof of the integrability of $Q_{a}$ is more involved than one would perhaps expect. 
First of all, since $P_{a_{t}} * G(n)$ is a monotone function of $t$, the kernel $\tilde{W} P_{a_{t}} * G(n)=\tilde{W} P_{a_{t}} * G(n)=e^{2 t}(d / d t) P_{a_{t}} * G(n)$ has constant sign; therefore, by Fubini's Theorem, the integrability of $Q_{a}$ will follow from the existence of the iterated integral

$$
\int_{\overline{\mathbb{a}} \alpha} \int_{\overline{\mathbb{Q}}_{2 \alpha}}\left(\tilde{W} P_{a} * G\right)(\exp (X+Y)) d X d Y
$$

(in this proof we systematically use $X, X^{\prime}$ and $Y, Y^{\prime}$ to denote elements of $\overline{\mathrm{g}}_{\alpha}$ and $\overline{\mathrm{g}}_{2 \alpha}$ respectively). Secondly, because of the homogeneity property (14) it is enough to consider the case $a=e=$ identity.

Now, if $n=\exp (X+Y)$ and $n^{\prime}=\exp \left(X^{\prime}+Y^{\prime}\right)$, then

$$
n^{\prime-1} n=\exp \left(X-X^{\prime}+Y-Y^{\prime}+\frac{1}{2}\left[X, X^{\prime}\right]\right),
$$

where $X-X^{\prime} \in \overline{\mathrm{g}}_{\alpha}$ and $Y-Y^{\prime}+\frac{1}{2}\left[X, X^{\prime}\right] \in \overline{\mathrm{g}}_{2 \alpha}$. We then have

$$
\begin{array}{rl}
\tilde{W} P_{e} * & G(|X|,|Y|) \\
& =\int_{\mathfrak{n}} \tilde{W} P_{e}\left(\left|X^{\prime}\right|,\left|Y^{\prime}\right|\right) G\left(\left|X-X^{\prime}\right|,\left|Y-Y^{\prime}+\frac{1}{2}\left[X, X^{\prime}\right]\right|\right) d X d Y
\end{array}
$$

where we write $F(|X|,|Y|)=F(\exp (X+Y))$ whenever $F$ is a biradial function of $\bar{N}$. Integrating over $\overline{\mathrm{g}}_{2 \alpha}$, and since the integrand in (16) has constant sign as a function of $Y$, we can exchange the order of the integrations over $\bar{g}_{2 \alpha}$ and $\bar{n}$ and get

$$
\begin{aligned}
\int_{\overline{\mathrm{d}}_{2 \alpha}} & \tilde{W} P_{e} * G(|X|,|Y|) d Y \\
& =\int_{\overline{\mathrm{n}}} \tilde{W} P_{e}\left(\left|X^{\prime}\right|,\left|Y^{\prime}\right|\right)\left(\int_{\overline{\mathrm{g}}_{2 \alpha}} G\left(\left|X-X^{\prime}\right|,\left|Y-Y^{\prime}+\frac{1}{2}\left[X, X^{\prime}\right]\right|\right) d Y\right) d X^{\prime} d Y^{\prime} \\
& =\int_{\overline{\mathrm{n}}} \tilde{W} P_{e}\left(\left|X^{\prime}\right|,\left|Y^{\prime}\right|\right)\left(\int_{\overline{\mathrm{g}}_{2 \alpha}} G\left(\left|X-X^{\prime}\right|,|Y|\right) d Y\right) d X^{\prime} d Y^{\prime}
\end{aligned}
$$

because of invariance under translations. Since

$$
G\left(\left|X-X^{\prime}\right|,|Y|\right)=\beta\left(\eta\left|X-X^{\prime}\right|^{4}+|Y|^{2}\right)^{-k},
$$

the change of variable $Y \rightarrow \eta^{1 / 2}\left|X-X^{\prime}\right|^{2} Y$ shows that

$$
\int_{\overline{\mathbb{B}}_{2 \alpha}} G\left(\left|X-X^{\prime}\right|,|Y|\right) d Y=\beta \eta^{1 / 2 m_{2 \alpha}-4 k}\left|X-X^{\prime}\right|^{2 m_{2 \alpha}-4 k} \int_{\overline{\mathfrak{d}}_{2 \alpha}}\left(1+|Y|^{2}\right)^{-k} d Y .
$$

Since under the assumption $m_{\alpha}+2 m_{2 \alpha}>2$ one has

$$
2 k=\frac{1}{2}\left(m_{\alpha}+2 m_{2 \alpha}-2\right)>m_{2 \alpha}=\operatorname{dim} \overline{\mathrm{g}}_{2 \alpha},
$$


the last integral exists, and we conclude that

$$
\begin{array}{rl}
\int_{\overline{\mathrm{Q}}_{2 \alpha}} \tilde{W} P_{2} & * G(|X|,|Y|) d Y \\
& =\mathrm{C}_{1} \int_{\overline{\tilde{n}}} \tilde{W} P_{e}\left(\left|X^{\prime}\right|,\left|Y^{\prime}\right|\right)\left|X-X^{\prime}\right|^{2 m_{2 \alpha}-4 k} d X^{\prime} d Y^{\prime}
\end{array}
$$

where $\mathbf{C}_{1}$ is a constant. Now, let $\Sigma=\left\{X \in \bar{g}_{\alpha}:|X|=1\right\}$ and introduce polar coordinates in $\bar{g}_{\alpha}: X^{\prime}=\rho \xi, 0 \leqslant \rho, \xi \in \Sigma$; since $2 m_{2 \alpha}-4 k=-m_{\alpha}+2$ the last integral becomes

$$
\int_{0}^{\infty} I(\rho)\left[\int_{\Sigma}|X-\rho \xi|^{-m_{\alpha}+2} d \xi\right] \rho^{m_{\alpha}-1} d \rho
$$

where $I(\rho)=\int_{\bar{g}_{2 \alpha}} \tilde{W} P_{e}(\rho,|Y|) d Y$. Now, consider the function

$$
X \rightarrow \int_{\Sigma}\left|\rho^{-1} X-\xi\right|^{-m_{\alpha}+2} d \xi
$$

it is continuous on $\overline{\mathrm{g}}_{\alpha}$, smooth and harmonic for $|X| \neq \rho$ (with respect to the Laplacian associated with the inner product $(\cdot, \cdot))$, invariant under the corresponding rotation group, equal to $\sigma=$ measure of $\Sigma$ for $X=0$, and not identically constant. One can therefore see that

$$
\int_{\Sigma}\left|\rho^{-1} X-\xi\right|^{-m_{\alpha}+2} d \xi= \begin{cases}\sigma & \text { for }|X| \leqslant \rho, \\ \sigma \rho^{m_{\alpha}-2}|X|^{-m_{\alpha}+2} & \text { for }|X| \geqslant \rho,\end{cases}
$$

which implies that (19) can be rewritten as

$$
\sigma|X|^{-m_{\alpha}+2} \int_{0}^{|X|} I(\rho) \rho^{m_{\alpha}-1} d \rho+\sigma \int_{|X|}^{\infty} I(\rho) \rho d \rho .
$$

In order to evaluate $I(\rho)$ we make use of the formula $[3$, p. 65]

$$
P_{a_{t}}(|X|,|Y|)=e^{-m t}\left[\left(e^{-2 t}+c|X|^{2}\right)^{2}+4 c|Y|^{2}\right]^{-m / 2}
$$

where $c=\left(4\left(m_{\alpha}+4 m_{2 \alpha}\right)\right)^{-1}, m=m_{\alpha}+2 m_{2 \alpha}$, which yields

$$
\begin{aligned}
I(\rho)= & \int_{\bar{d}_{2 \alpha}} \tilde{W} P_{e}(\rho,|Y|) d Y \\
=-m \int_{\overline{\mathrm{g}}_{2 \alpha}}\left[\left(1+c \rho^{2}\right)^{2}\right. & \left.+4 c|Y|^{2}\right]^{-m / 2-1} \\
& \times\left[\left(1+c \rho^{2}\right)^{2}+4 c|Y|^{2}-2\left(1+c \rho^{2}\right)\right] d Y .
\end{aligned}
$$

After the change of variable $Y \rightarrow\left(1+c \rho^{2}\right) Y$, this becomes 


$$
I(\rho)=\text { const } \cdot\left[2 b\left(1+c \rho^{2}\right)^{-\left(m_{\alpha}+m_{2 \alpha}+1\right)}-\left(1+c \rho^{2}\right)^{-\left(m_{\alpha}+m_{2 \alpha}\right)}\right]
$$

where

$$
b=\left[\int_{\overline{\mathrm{g}}_{2 \alpha}}\left(1+4 c|Y|^{2}\right)^{-m / 2+1} d Y\right]^{-1} \int_{\overline{\mathrm{g}}_{2 \alpha}}\left(1+4 c|Y|^{2}\right)^{-m / 2} d Y .
$$

Evaluating these two integrals gives $b=m^{-1}\left(m_{\alpha}+m_{2 \alpha}\right)$. Now, substituting this expression for $I(\rho)$ into (20), one checks by differentiation that (20) equals $\mathbf{C}\left(1+c|X|^{2}\right)^{-\left(m_{\alpha}+m_{2 \alpha}-1\right)}$, where the constant $\mathbf{C}$ is given by $\mathbf{C}=\sigma\left(m_{\alpha}-2\right)$ $\cdot\left[2 \mathrm{~cm}\left(m_{\alpha}+m_{2 \alpha}-1\right)\right]^{-1}$. Therefore,

$$
\int_{\bar{d}_{2 \alpha}} \tilde{W} P_{e} * G(|X|,|Y|) d Y=\text { const } \cdot\left(1+c|X|^{2}\right)^{-\left(m_{\alpha}+m_{2 \alpha}-1\right)} .
$$

Since $2\left(m_{\alpha}+m_{2 \alpha}-1\right)>m_{\alpha}=\operatorname{dim} \overline{\mathfrak{g}}_{\alpha}$, the above expression is integrable over $\overline{\mathrm{g}}_{\alpha}$; we have therefore shown that (15) exists, so that $\tilde{W} P_{a} * G$ is integrable. Now choose $c$ so that $Q_{e}=c \tilde{W} P_{e}^{*} G$ has integral equal to one; then $Q_{e}>0$ and by the homogeneity property (14) one has

$$
Q_{a}(n)=e^{m a(\log a)} Q_{e}\left(a^{-1} n a\right)
$$

so that $Q_{a}>0$ for all $a \in A$. This finishes the proof of (i).

Integrating (22) and recalling that the Jacobian of the change of variable $n \rightarrow a n a^{-1}$ is $e^{-m a(\log a)}$, one gets

$$
\int_{N} Q_{a}(n) d n=e^{m \alpha(\log a)} \int_{N} Q_{e}\left(a^{-1} n a\right) d n=\int_{N} Q_{e}(n) d n=1,
$$

proving (ii). On the other hand, letting $a=a_{t}$ in (22) and integrating over $\bar{N}(\varepsilon)$ one gets

$$
\int_{\overline{N(\varepsilon)}} Q_{a_{t}}(n) d n=e^{m t} \int_{\bar{N}(\varepsilon)} Q_{e}\left(a_{t}^{-1} n a_{t}\right) d n=\int_{a_{t}^{-1} \bar{N}(\varepsilon) a_{t}} Q_{e}(n) d n .
$$

Since $\left\|a_{t}^{-1} n a_{t}\right\|=e^{4 t}\|n\|$, it follows that $a_{t}^{-1} \bar{N}(\varepsilon) a_{t}=\bar{N}\left(e^{4 t} \varepsilon\right)$. Therefore, as $t \rightarrow+\infty$, the last integral converges to $\int_{N} Q_{e}(n) d n=1$. This shows (iii) and finishes the proof of the lemma.

ReMARK. Although the kernel $Q_{a}$ is not an elementary function in general, formula (21) gives its integral over $\bar{g}_{2 \alpha}$. In particular, when $\bar{g}_{2 \alpha}=(0)$ (case of real hyperbolic space) one gets the explicit expression

$$
Q_{a_{t}}(X)=\mathbf{C} e^{\left(m_{\alpha}-2\right) t} /\left(e^{2 t}+|X|^{2}\right)^{m_{\alpha}-1} .
$$

Now, for each function $f$ on $\bar{N}$ for which the following convolutions make sense, define a 1-form $\omega_{f}$ on $M$ by 


$$
\begin{aligned}
& \omega_{f}(\tilde{W})_{n a \cdot o}=f * Q_{a}(n), \\
& \omega_{f}(\tilde{X})_{n a \cdot o}=f * P_{a} * r_{X}(n) \quad(X \in \overline{\mathfrak{n}}) .
\end{aligned}
$$

As before, all convolutions are taken on the group $\bar{N}$. Note that, if $f$ is sufficiently nice, then $f * P * G$ exists and $\omega_{f}$ is just the exterior derivative of this function; but this convolution is not in general defined for $f \in L^{p}(\bar{N})$ and $p$ in the full range $1<p<m$.

THEOREM 3. Let $1<p<m$ and let $f \in L^{p}(\bar{N})$. Then

(i) $\omega_{f}$ is well defined and belongs to $\mathbf{H}^{p}$,

(ii) $\omega_{f}(\tilde{W})$ converges in $L^{p}$ and a.e. to $f$,

(iii) for each $X \in \overline{\mathrm{g}}_{\alpha}\left(\right.$ resp. $\left.X \in \overline{\mathrm{g}}_{2 \alpha}\right), \omega_{f}(\tilde{X})$ converges in $L^{q}\left(\right.$ resp. $\left.L^{p}\right)$ and a.e. to the Riesz transform $R_{X} f=f * r_{X}$.

Proof. For $f \in L^{p}(\bar{N})$, the convolutions $f * P$ and $f * Q$ are smooth functions on $M$ which are uniformly in $L^{p}(\bar{N})$. Since the Riesz transforms are defined in $L^{p}(\bar{N})$, it follows that $\omega_{f}$ is a well-defined differential form on $M$.

Now notice that if $f(n)$ and $F(n a \cdot o)=F_{a}(n)$ are functions on $\bar{N}$ and $M$, respectively, then derivatives of the convolution $f * F_{a}$ with respect to the vector fields $\tilde{X}$ satisfy $\tilde{X}\left(f * F_{a}\right)=f * X F_{a}$. Therefore, for $X, Y \in \overline{\mathfrak{n}}$ one has

$$
\tilde{X} \omega_{f}(\tilde{Y})-\tilde{Y} \omega_{f}(\tilde{X})=f * P *\left(X r_{Y}-Y r_{X}\right)=f * P * r_{[X Y]}=\omega_{f}([\tilde{X}, \tilde{Y}]) .
$$

Also, since $\tilde{X}$ and $\tilde{W}$ commute, $\tilde{X} \omega_{f}(\tilde{W})-\tilde{W} \omega_{f}(\tilde{X})=0=\omega_{f}([\tilde{X} \tilde{W}])$. Since the vector fields $\tilde{X}, \tilde{W}(X \in \overline{\mathfrak{n}})$ span the tangent space to $M$ at every point, it follows that $\omega_{f}$ is closed. In order to show that it is coclosed, we may assume that $f$ is, say, continuous of compact support, the result for a general $f \in L^{p}(\bar{N})$ following by a standard approximation argument. Then $\delta \omega_{f}$ $=\delta d(f * P * G)=\Delta(f * P * G)$, where $\Delta$ is the Laplace-Beltrami operator on $M$; since $P$ and $G$ are both biradial, the function $f * P * G=f * G * P$ is harmonic, and so is annihilated by $\Delta$.

The Poisson integral of $f$ is uniformly in $L^{p}(N)$ and it converges in $L^{p}$ to its boundary value $f$. Since convolution against the Riesz kernels $r_{X}\left(X \in \overline{\mathrm{g}}_{\alpha}\right)$ is a bounded operator from $L^{p}(\bar{N})$ into $L^{q}(\bar{N})$, one concludes that the function $\omega_{f}(\tilde{X})$ (which, we recall, is not always harmonic) is uniformly in $L^{q}(N)$ and that it converges to $R_{X} f=f * r_{X}$ in $L^{q}$-norm. The same argument applies to the components $\omega(\tilde{X})$ when $X \in \bar{g}_{2 \alpha}$, giving a bounded operator from $L^{p}$ into $L^{p}$. If $X_{1}, \ldots, X_{n}$ is an orthonormal basis of $\overline{\mathrm{g}}_{\alpha}, \sum X_{i} r_{X_{i}}$ is the Diracdistribution on $\bar{N}$; therefore $\sum_{i} \tilde{X}_{i} \omega\left(\tilde{X}_{i}\right)=\sum f * X_{i}\left(P * r_{X_{i}}\right)=f * P$ and so this function is also uniformly in $L^{p}(\bar{N})$. Finally, $Q$ being an approximate identity, the component $\omega_{f}(\tilde{W})=f * Q$ is clearly uniformly in $L^{p}(\bar{N})$ and it converges in $L^{p}$-norm to $f$. Q.E.D. 
In the next theorem we show that the mapping $f \rightarrow \omega_{f}$ from $L^{p}(\bar{N})$ into $\mathbf{H}^{p}$ is actually an isomorphism onto. Together with Theorem 3 this implies that every form $\omega$ in $\mathbf{H}^{p}$ has boundary values in the appropriate sense, and that the boundary values of the components of $\omega$ along the vector fields $\tilde{X}(X \in \overline{\mathfrak{n}})$ are precisely the Riesz transforms of the boundary value of the component of $\omega$ along the vector field $\tilde{W}$.

THEOREM 4. Let $\omega \in \mathbf{H}^{p}$. Then there exists a unique $f \in L^{p}(\bar{N})$ such that $\omega=\omega_{f}$.

Proof. Let $X_{1}, \ldots, X_{m_{\alpha}}$ be an orthonormal basis of $\overline{\mathrm{g}}_{\alpha}, \tilde{L}=\sum \tilde{X}_{i}^{2}$, and let $Y$ be in the center of $\overline{\mathfrak{n}}$. For any closed form $\xi$ on $M$, a straightforward computation using Lemma 1 shows that $\Delta\left(\sum_{i} \tilde{X}_{i} \xi\left(\tilde{X}_{i}\right)\right)=\tilde{L} \delta \xi$ and $\Delta \xi(\tilde{Y})$ $=\tilde{Y} \delta \xi$. Therefore $\omega \in \mathbf{H}^{p}$ implies that the functions $\sum \tilde{X}_{i} \omega\left(\tilde{X}_{i}\right)$ and $\omega(\tilde{Y})$ are harmonic; they can then be written as Poisson integrals $\sum_{i} \tilde{X}_{i} \omega\left(\tilde{X}_{i}\right)=f * P$ and $\omega(\tilde{Y})=\varphi * P$, with $f, \varphi \in L^{p}(\bar{N})$. Let $\omega_{f}$ be the form associated to $f$ by equation (23); then $\omega_{f}(\tilde{Y})=f * P * r_{Y}=\sum \tilde{X}_{i} \omega\left(\tilde{X}_{i}\right) * Y G$, and since $Y$ is in the center of $\overline{\mathfrak{n}}$, this last expression is equal to $\tilde{Y}\left(\sum_{i} \tilde{X}_{i} \omega\left(\tilde{X}_{i}\right)\right) * G$. But $\tilde{Y}\left(\sum \tilde{X}_{i} \omega\left(\tilde{X}_{i}\right)\right)=\tilde{L} \omega(\tilde{Y})$, so we can write

$$
\omega_{f}(\tilde{Y})=\tilde{L} \omega(\tilde{Y}) * G=\tilde{L}(\varphi * P) * G=\varphi * \tilde{L} P * G .
$$

Now the functions $G, P$ and $\tilde{L} P$ are biradial and since $G$ is a fundamental solution for the operator $L$, one has

$$
\tilde{L} P * G=G * \tilde{L} P=\tilde{L}(G * P)=\tilde{L}(P * G)=P .
$$

Substituting in (24) we get $\omega_{f}(\tilde{Y})=\varphi * P=\omega(\tilde{Y})$.

Now set $\omega^{\prime}=\omega-\omega_{f}$; then $\omega^{\prime} \in \mathbf{H}^{p}$ and if $Y$ is in the center of $\bar{n}$, we have shown above that $\omega^{\prime}(\tilde{Y})=0$; let $X$ be an arbitrary element of $\bar{n}$; then

$$
\tilde{Y} \omega^{\prime}(\tilde{X})=\tilde{X} \omega^{\prime}(\tilde{Y})+\omega^{\prime}([\tilde{Y}, \tilde{X}])=0 .
$$

Consequently, the functions $\omega^{\prime}(\tilde{X})$ are constant on the orbits of the center of $\bar{N}$ under the action $\tau$; on the other hand, $\omega^{\prime} \in \mathbf{H}^{p}$ implies that $\omega^{\prime}(\tilde{X})$ is in $L^{p}$ of the orbits of $\tau(\bar{N})$, so that these functions cannot be constant on the submanifolds $\tau\left(\exp \bar{g}_{2 \alpha}\right)$ unless they are identically zero. We then conclude that $\omega^{\prime}(\tilde{X})=0$ for all $X \in \bar{n}$, and exactly the same argument shows that $\omega^{\prime}(\tilde{W})=0$. Thus $\omega^{\prime} \equiv 0$, proving that $\omega=\omega_{f}$, as claimed.

Theorems 3 and 4 can be summarized by

Corollary. Let $\omega \in \mathbf{H}^{p}$; then $\omega(\tilde{W}), \omega(\tilde{X}), \omega(\tilde{Y})$ have boundary values in $L^{p}, L^{q}$, and $L^{p}$ respectively, and if $f=$ boundary value of $\omega(\tilde{W})$, then $\omega=\omega_{f}$.

ReMARK. As observed before, the results of $\$ \S 2-3$ hold under the assumption $m=m_{\alpha}+2 m_{2 \alpha} \geqslant 3$. In the two remaining cases $m=1$ (usual upper- 
half plane) and $m=2$ (three-dimensional real hyperbolic space), appropriate modifications in the definitions of the Riesz transforms and in the length of the vector field $\tilde{W}$, yield similar results. In particular, for $m=1$, one gets the classical relation between conjugate harmonic functions in the upper-half plane and the Hilbert transform on the line.

\section{REFERENCES}

1. R. R. Coifman and G. Weiss, Invariant systems of conjugate harmonic functions associated with compact Lie groups, Studia Math. 44 (1972), 301-308. MR 49 \#5736.

2. G. B. Folland, $A$ fundamental solution for a subelliptic operator, Bull. Amer. Math. Soc. 79 (1973), 373-376. MR 47 \#3816.

3. S. Helgason, $A$ duality for symmetric spaces with applications to group representations, Advances in Math. 5 (1970), 1-154. MR 41 \#8587.

4. L. Hörmander, Hypoelliptic second order differential equations, Acta Math. 119 (1967), 147-171. MR 36 \#5526.

5. A. Korányi, Boundary behavior of Poisson integrals on symmetric spaces, Trans. Amer. Math. Soc. 140 (1969), 393-409. MR 39 \# 7132.

6. A. Korányi and S. Vági, Singular integrals on homogeneous spaces and some problems of classical analysis, Ann. Scuola Norm. Sup. Pisa 25 (1971).

7. - Cauchy-Szegö integrals for systems of harmonic functions, Ann. Scuola Norm. Sup. Pisa 26 (1972), 181-196.

8. E. M. Stein, Singular integrals and estimates for the Cauchy-Riemann equations, Bull. Amer. Math. Soc. 79 (1973), 440-445. MR 47 \#3851.

9. E. M. Stein and G. Weiss, On the theory of harmonic functions of several variables. I: The

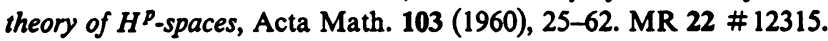

Department of Mathematics, University of Massachusetts, Amherst, Massachusetts 01002 (Current address of Aroldo Kaplan)

Department of Mathematics, Belfer Graduate School of Science, Yeshiva University, NEW YORK, NEW YORK 10033

Current address (Robert Putz): Department of Mathematics, New York Community College, City University of New York, Brooklyn, New York 11201 